previous history of difficult airway management with postponement of this procedure 3 days before. After patient's consent, we succefully managed the case with an ultrasoundguided IPB and a PIFB (figure 2), total local anaesthetic solution - $20 \mathrm{~mL}$ of mepivacaine $1,5 \%$ \& ropivacaine $0,375 \%$ under light sedation. The procedure was uneventful, for patient and surgical team.

Conclusions IPB and PIFB successfully managed anaesthesia \& analgesia of this case under light sedation, avoiding GA and management of a documented difficult airway patient. Latest evidence supports IPB as a good technique for BCS(2) and PIFB is a relatively novel block suited for inner quadrant breast surgery approach(3). RA has been a good alternative avoiding airway management on this documented difficult airway, provided excellent surgery conditions, pain relief and a high level of patient satisfaction.

Approval has been granted by the ethics committee

\section{PTERYGOPALATINE BLOCKADE IN THE ALGORITHM OF TREATMENT OF OPHTHALMIC COMPLICATIONS OF HERPES ZOSTER}

I Oleshchenko*, T lureva. Irkutsk Branch of S.N. Fyodorov 'Eye Microsurgery' Federal State Institution, Irkutsk, Russian Federation

\subsection{6/rapm-2021-ESRA.171}

Background and Aims Ophthalmoplegia in herpes Zoster is quite rare. At the same time, we observed a case of relapse of herpes Zoster with the clinic of ptosis of the upper eyelid on the background of a pronounced pain syndrome without vesicular rashes as the primary clinical signs. Relief of pain syndrome against the background of antiviral therapy is one of the primary tasks. To evaluate the effectiveness of pterygopalatine blockade (PPB) in the algorithm of treatment of atypical ophthalmic herpes Zoster with severe pain syndrome and ophthalmoplegia.

Methods Patient 60 years old. Complaints about OS blepharoptosis, pain (VAS = 4). The edge of the OS eyelid is lowered to the upper edge of the pupil, the cornea is transparent, there are no signs of uveitis. The condition is regarded as ganglionitis n.ophtalmicus of unknown etiology. The patient underwent PPB (4 mL ropivacaine $0.5 \%$ ) for pain relief in herpetic ophthalmic neuralgia

Results 40 minutes after $\mathrm{PPB}$, the pain was relieved, VAS = 0. 2 hours after PPB, the ptosis decreased. After 72 hours the patient had a draining herpetic rash on the skin in the projection of the III branch of the trigeminal nerve on the left. Further treatment of the patient was continued by an infectious disease specialist.

Conclusions The experience gained in the use of the PPB in the algorithm for the treatment of ophthalmic complications of herpes Zoster allowed us to achieve not only the relief of pain, but also to reduce the severity of the inflammatory reaction of extraocular muscles, ptosis.

\section{ULTRASOUND GUIDED COMBINED SCIATIC PLUS FEMORAL NERVE BLOCK IN HIGH RISK PATIENTS POSTED FOR LOWER LIMB SURGERIES}

N Adhyapak*, R Mane, M Patil, R Kerur, P Apoorva, A Qadir Zeeshan. KAHER's I N Medical College, Belagavi, India
Background and Aims Regional Nerve Blocks are now the mainstay for high risk patients undergoing surgery where General, Spinal or Epidural Anaesthesia have poor outcome. Combined Sciatic and Femoral Nerve block given for lower limb surgeries like amputation, arthroplasty, ORIF in patients having multiple co-morbidities like Ishaemic Heart Disease (IHD), COPD, Uncontrolled DM and HTN. Ultrasound guided nerve blocks are effective, safe and have reduced incidence of neurological damage and Local Anaesthetic Systemic Toxicity (LAST).

Methods 5 patients undergoing lower limb surgery, in KLE's Dr. Prabhakar Kore Charitable Hospital and MRC were considered. Three of the patients had IHD with EF 40\%. One had RV Dysfunction with IVC collapsibility $<50 \%$. Another had COPD and was in sepsis. A complete pre-anaesthetic evaluation was done after selecting appropriate patients. Under ultrasound guidance, with patient in sciatic and femoral nerve blocks were given with equal mixtures of $0.5 \%$ Bupivacaine + $2 \%$ Lignocaine $+2 \mathrm{ml}$ of Soda Bicarbonate. Intra-operatively hemodynamics was maintained. Case 1 required rescue Epidural Anaesthesia. For case 3 i.v paracetamol was given for additional analgesia. In rest of the cases no additional analgesia/anaesthesia was needed.

Results

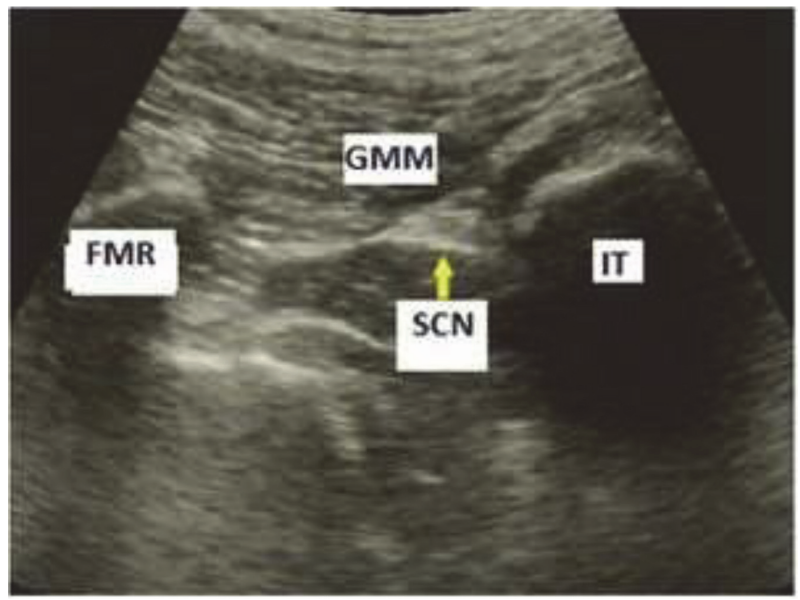

Abstract 172 Figure 1

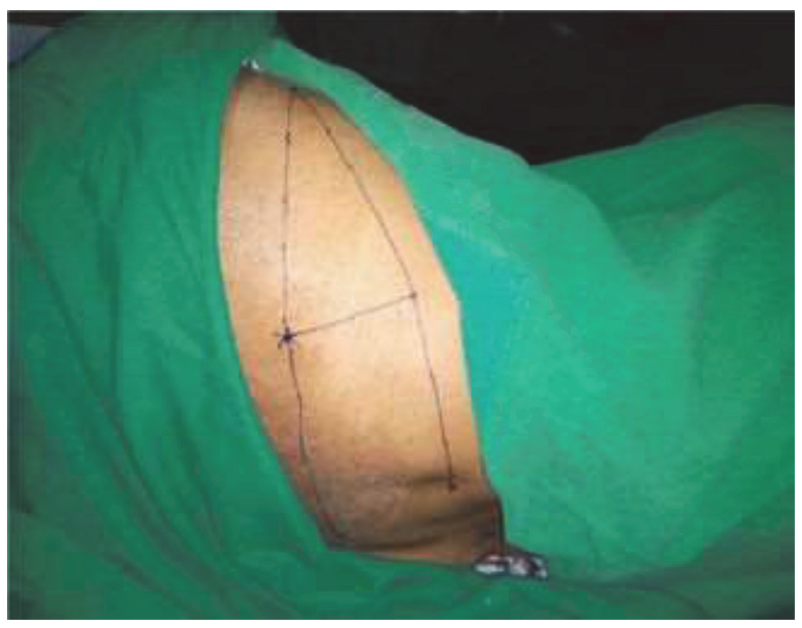

Abstract 172 Figure 2 




Abstract 172 Figure 3

- Conclusions Combined Sciatic and Femoral Nerve block is an effective and viable alternative to either General, Spinal or Epidural Anaesthesia as it provides better hemodynamic stability and good post operative analgesia in high risk patients without resulting in any adverse incidents.

\section{PERIPHERAL NERVE BLOCKS ALLOW HIP FRACTURE SURGICAL MANAGEMENT IN AN EXTREMELY FRAGILE PATIENT: NO PENG NO GAME}

F Gargano, A Strumia*, F Costa, G Pascarella, S Rizzo, V Antinolfi, G Biondo, G Rizzello, FE Agrò. Università Campus Biomedico di Roma, Rome, Italy

\subsection{6/rapm-2021-ESRA.173}

Background and Aims Hip fracture represents a frequent cause of disability in the elderly [1]. Early surgery $(<48 \mathrm{~h})$ has been proven to be associated with lower risk of mortality and morbidity [2]. Regional anaesthesia seems to be the best option, but there is no consensus when a central block is not indicated [3]. We present a case of a multimorbidity patient under anticoagulant drug, underwent hip fracture surgical repair with peripheral nerve blocks.

Methods Female 84 y.o. Atrial fibrillation, PMK wearer, hypertension, renal failure, CODP, cerebrovascular disease, OSAS, confusion and psychomotor agitation.

Echocardiogram: severe systolic function reduction, estimated election fraction $23 \%$.

Right sub-capital hip fracture, candidates for hip endoprosthesis.

Anticoagulant therapy with apixaban with no data on last assumption; thromboelastogram showed augmented values, so a central block was not indicated. Patient's multimorbidity highly elevated the risk of general anaesthesia.

With patient consent, peripheral blocks and sedation were considered. Ultrasound guided Pericapsular Nerve Group Block (PENG) was performed (ropivacaine 0,5\% $20 \mathrm{ml}+$ dexamethasone $4 \mathrm{mg}$ ), to block femoral, obturator and accessory obturator nerves, which innervate the hip [4]. Lateral femoral cutaneous nerve was also blocked, to cover surgical incision. Moderate sedation was given, maintaining spontaneous breath. Results Surgical procedure was carried out uneventfully and without pain, parameters were good and stable during and after surgery.

Conclusions Regional anaesthesia could be the key in fragile patients. Knowledge of anatomy and different techniques is

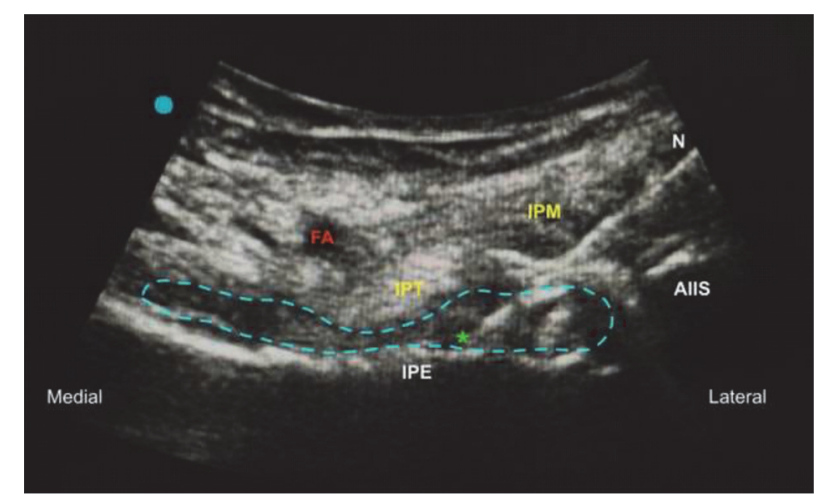

Abstract 173 Figure 1

$\mathrm{FA}=$ femoral artery; IPM=iliopsoas muscle; IPT=ilipsoas tendon; $\mathrm{N}=$ needle; IPE=iliopubic eminence; AllS=anterior inferior iliac spine.

fundamental to tailor the anaesthetic plan on patient conditions and surgical approaches.

\section{PERIOPERATIVE JOURNEY OF PATIENTS HAVING UPPER LIMB NERVE BLOCKS- A SERVICE EVALUATION AND QI PROJECT}

${ }^{1} \mathrm{M}$ Singh* ${ }^{2}{ }^{2} \mathrm{~S}$ Dondapati, ${ }^{2} \mathrm{~A}$ Singh. ${ }^{1}$ Stoke School of Anaesthesia, Stoke-on-Trent, UK; ${ }^{2}$ New Cross Hospital, Wolverhampton, UK

\subsection{6/rapm-2021-ESRA. 174}

Background and Aims Regional anaesthesia (RA) improves postoperative analgesia and can contribute to a reduction in postoperative nausea and vomiting (PONV). RA can improve value of care by reducing length of stay and enhancing patient satisfaction $^{1}$.

Regional nerve blocks are commonly performed for upper limb day case surgeries at our centre. We did a prospective patient survey on perioperative experience of upper limb surgery with nerve blocks.

Methods Patients who had regional nerve blocks for upper limb surgery were given proforma to fill after surgery with pre-stamped envelopes. We included 28 proformas returned from March to June 2019 for the survey.

Results All patients received anaesthetic information pre-surgery which included pre-assessment nurse (7/28), information leaflet $(2 / 28)$ and both (19/28). One patient found information provided unsatisfactory. Quarter of (8/28) patients reported severe pain and PONV during first 24 hours and timings of first oral pain relief was variable with non-standardised analgesics on prescription.

Most of patients (92\%) would prefer to have nerve block again.

Conclusions Results showed that post op pain prescription varied. Although most patients were satisfied with nerve block information but could be improved. We recommended to standardise post-operative discharge prescription. We suggested to produce patient video about the perioperative journey of nerve blocks for upper limb surgery which has been produced and will be implemented soon as delayed by pandemic.

Regular patient satisfaction surveys are needed for service evaluation and improvement. 